\title{
Reliability of freehand three-dimensional ultrasound to measure scapular rotations
}

\author{
Lynn A. Worobey, PhD; ${ }^{1-3}$ Ima A. Udofa, BS; ${ }^{1-2}$ Yen-Sheng Lin, PhD; ${ }^{1,4}$ Alicia M. Koontz, PhD; ${ }^{1-2,4}$ Shawn S. \\ Farrokhi, DPT, PhD; ${ }^{2,5}$ Michael L. Boninger, $\mathrm{MD}^{1-4^{*}}$ \\ ${ }^{1}$ Human Engineering Research Laboratories, Department of Veterans Affairs Pittsburgh Healthcare System, Pitts- \\ burgh, PA; Departments of ${ }^{2}$ Bioengineering, ${ }^{3}$ Physical Medicine and Rehabilitation, ${ }^{4}$ Rehabilitation Sciences and \\ Technology, and ${ }^{5}$ Physical Therapy, University of Pittsburgh, Pittsburgh, PA
}

\begin{abstract}
The objective of this study was to evaluate the reliability of using freehand three-dimensional ultrasound to measure scapular rotations (internal/external, upward/downward, anterior/posterior). The scapular position in 22 healthy, nondisabled individuals was imaged three times in four testing positions of interest (arm at rest and humeral elevation in the sagittal, frontal, and scapular planes). We found substantial reliability across scanning positions and scapular rotations, with intraclass correlation coefficients ranging from 0.62 to 0.95 . The highest reliability was found in the rest testing position. Our standard error of measurement was less than 2 degrees for all measurements and less than 0.5 degrees for most. Minimum detectable change ranged from 0.37 to 3.08 degrees. Our results agree with the pattern of movement found in other studies, with the scapula moving toward a more externally rotated, upwardly rotated, and posteriorly tilted position with humeral elevation. Further study is warranted to compare our methods to a gold standard, apply them to evaluation of dynamic movement, and determine whether they can be used to detect shoulder pathology.
\end{abstract}

Key words: anterior/posterior tilting, freehand ultrasound, humeral elevation, internal/external rotation, minimum detectable change, reliability, rotation, scapula, standard error of measurement, upward/downward rotation.

\section{INTRODUCTION}

As the base of the shoulder, the scapula is integral to normal function of the joint. Altered scapular kinematics have been linked to shoulder pathology and impingement syndrome [1-5]. Attempts have been made to move beyond visual observation of shoulder dyskinesis, or altered scapular movement, to objective methods for detecting acute changes in movement. The gold standard for evaluating scapular movement has become bone pins. While highly accurate, this method is invasive and studies are generally limited to very small sample sizes [6-7]. Radiography and fluoroscopy have been used to visualize movement, but studies are either limited to one dimension of imaging or have limited testing time to minimize exposure to radiation. Magnetic resonance imaging allows for three-dimensional (3D) reconstruction of the

\footnotetext{
Abbreviations: $2 \mathrm{D}=$ two-dimensional, $3 \mathrm{D}=$ three-dimensional, ICC $=$ intraclass correlation coefficient, $\mathrm{MDC}=$ minimum detectable change, RMSE = root-mean-square error, SEM = standard error of measurement, VA = Department of Veterans Affairs.

*Address all correspondence to Michael L. Boninger, MD; VA Pittsburgh Healthcare System-Human Engineering Research Laboratories, 6425 Penn Ave, Suite 400, Pittsburgh, PA 15206; 412-822-3687; fax: 412-822-3699.

Email: boninger@pitt.edu

http://dx.doi.org/10.1682/JRRD.2014.01.0006
} 
scapula, but it is expensive and time consuming and does not allow for the evaluation of functional movement. Skin-based marker systems, which do not expose subjects to radiation and allow for evaluation of dynamic postures, have employed a variety of markers [3,7-12]. Unfortunately, inherent to the scapula gliding under skin and muscle during movement, skin-based systems are subject to error, particularly at higher angles of arm elevation $[7,13]$. Digitization techniques have also been used to characterize scapula movement; however, they are limited to static postures and are affected by the amount of skin, muscle, and adipose tissue between the bone and digitizer [13]. Additionally, previous studies vary in terms of which arm elevation planes are examined (sagittal, frontal, and scapular) and differences in technique make comparison across studies difficult.

On the basis of the limitations of previous studies, we proposed the novel application of freehand 3D ultrasound to evaluate scapular movement. Freehand ultrasound pairs two-dimensional (2D) ultrasound with motion capture to create a $3 \mathrm{D}$ data set. This method involves no radiation, is comparatively low cost, and allows for direct visualization of the bone. In the last $10 \mathrm{yr}$, the prevalence of using freehand 3D ultrasound to image soft tissue and bone has increased. Investigators have combined ultrasound and motion capture systems to image Achilles tendon strain [14-16], muscle architecture [17-18], muscle volume [19], and patellar movement [20-21]. To our knowledge, no previous studies have used ultrasound to evaluate scapular positioning.

The main objective of this study was to evaluate the reliability of using freehand $3 \mathrm{D}$ ultrasound to measure scapular rotations with the arm held in different static positions (arm at rest and elevated in the sagittal, frontal, and scapular planes). Secondary goals included establishing the reliability of our freehand system and manual point selection methods to identify the scapular border in ultrasound images.

\section{METHODS}

\section{Freehand Three-Dimensional Ultrasound System}

Ultrasound imaging was completed using a Philips HD11XE ultrasound machine equipped with a 5-12 MHz linear transducer (Philips Medical Systems; Bothell, Washington). This machine was limited to $6 \mathrm{~s}$ of video recording and trials were approximately $1 \mathrm{~min}$ in length, so an Epiphan Frame Grabber (10 Hz, Epiphan Systems;
Ottawa, Ontario, Canada) was used to record video collected during the trial. In order to track movement, the ultrasound probe was fitted with a custom orthogonal attachment equipped with Vicon markers (Vicon Motion Systems; Centennial, Colorado) (Figure 1(a)). Movement was recorded using Vicon Nexus software (Vicon Motion Systems) and 10 cameras (Vicon Motion Systems). Vicon cameras were arranged to maximize visibility of subject makers as well as the ultrasound probe. Vicon data were collected at a sampling frequency of $120 \mathrm{~Hz}$ using Nexus 1.8 software (Vicon Motion Systems). We determined the relationship between the ultrasound image and custom orthogonal attachment by collecting a static trial with additional Vicon markers in line with the beam of the probe and the edges of the scanning surface (Figure 1(b)). Before subject testing, our system was calibrated by scanning a phantom of known dimensions, yielding a root-mean-square error of (RMSE) $0.1 \mathrm{~cm}$.

\section{Participants}

Subjects were recruited through flyers and word of mouth at the Human Engineering Research Laboratories (Pittsburgh, Pennsylvania). Subjects were eligible to participate in this study if they were over the age of $18 \mathrm{yr}$, spoke English, and were able to raise their arm above their head. Subjects were excluded from this study if they had a history of fractures or dislocations in the shoulder from which they had not fully recovered; had upper-limb impairment, weakness, or spasticity that prevented smooth movement; or could not complete reach tasks while seated with support straps around the trunk.

\section{Testing Setup}

A testing chair was designed to isolate scapular movement and provide repeatable movements of the subject's dominant arm in space (Figure 2). The backrest of the chair was height adjustable to accommodate a multitude of torso lengths. An adjustable pad (height and depth) was also placed behind the contralateral shoulder to provide support. The location where the contralateral shoulder met the pad was marked on each subject so that the subject's posture could be readjusted if he or she shifted between trials. Additionally, the contralateral shoulder was held in place by a strap that reached from the shoulder pad, around the anterior portion of the shoulder, and down to the seat of the chair. An adjustable strap was also placed around the subject's trunk to minimize trunk movement (Figure 2(a)). Through the use of 


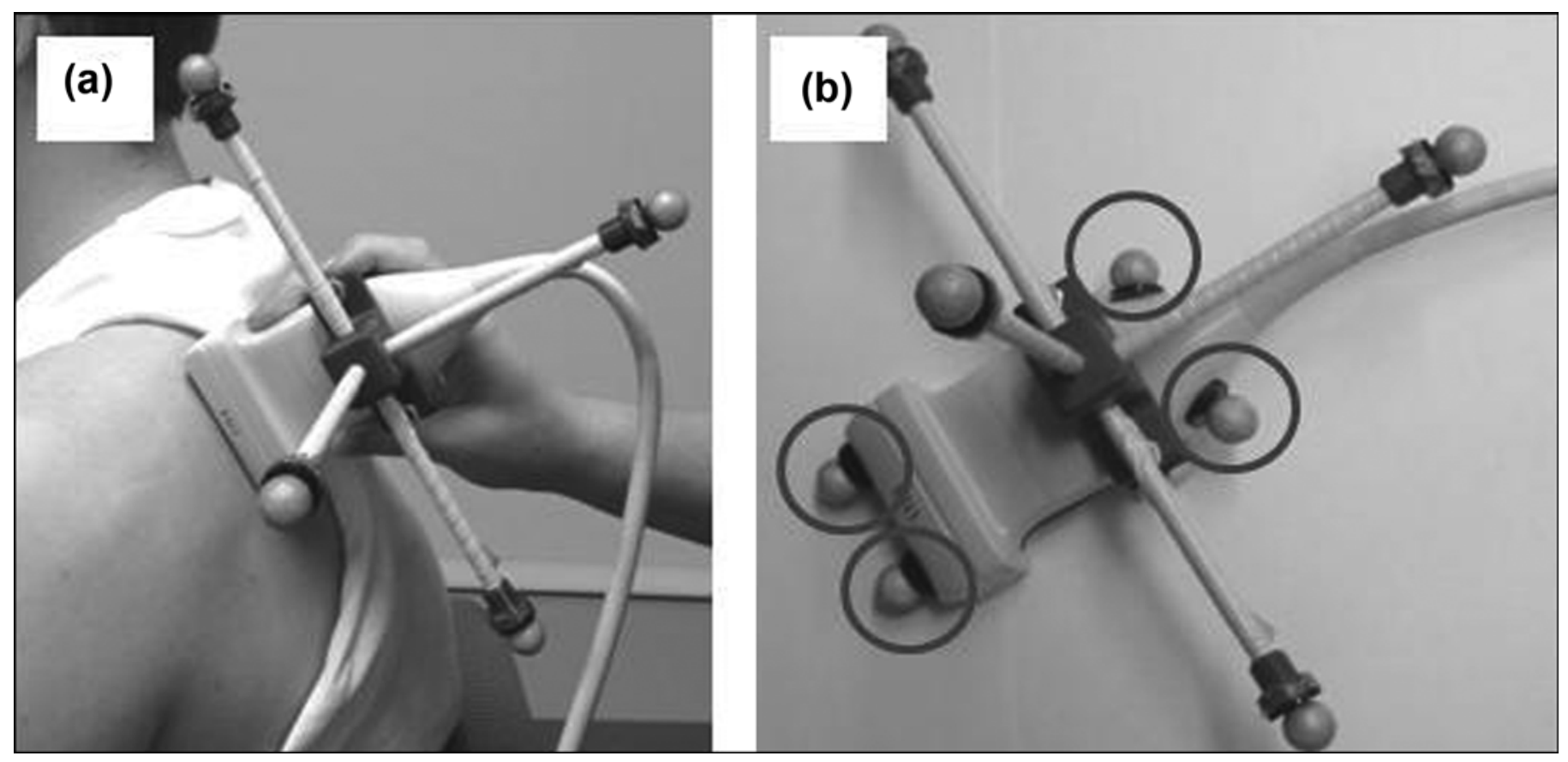

Figure 1.

(a) Ultrasound probe fitted with custom orthogonal attachment and Vicon markers and (b) additional markers added during static calibration to determine relationship between ultrasound image and custom attachment.

an angle-adjustable guide bar, the testing chair helped ensure consistency in holding the arm at the three elevated testing positions (Figure 2(b)).

For the first elevation trial, the subject raised his or her arm while maintaining contact with the guide bar until the angle of humeral elevation registered $120^{\circ}$ on a goniometer. A stop marker was placed on the guide bar and remained at this height for all elevation trials for that subject (Figure 2(c)). During testing, the dominant hand was against the guide bar with the thumb pointed upward. Participants were instructed to keep their head facing forward during the trial.

Participants wore a white tank top or removed their shirt to allow for placement of Vicon markers on the trunk and dominant arm. Marker placement followed the International Society of Biomechanics recommendations [22] and included the following bony landmarks: cervical 7 , thoracic 8 , sternum, xiphoid, acromion, lateral epicondyle, and medial epicondyle. A triad of markers was also placed on the upper arm in case of marker dropout. These markers allowed measurement of trunk and upper-arm movement during trials. Because scapular position would

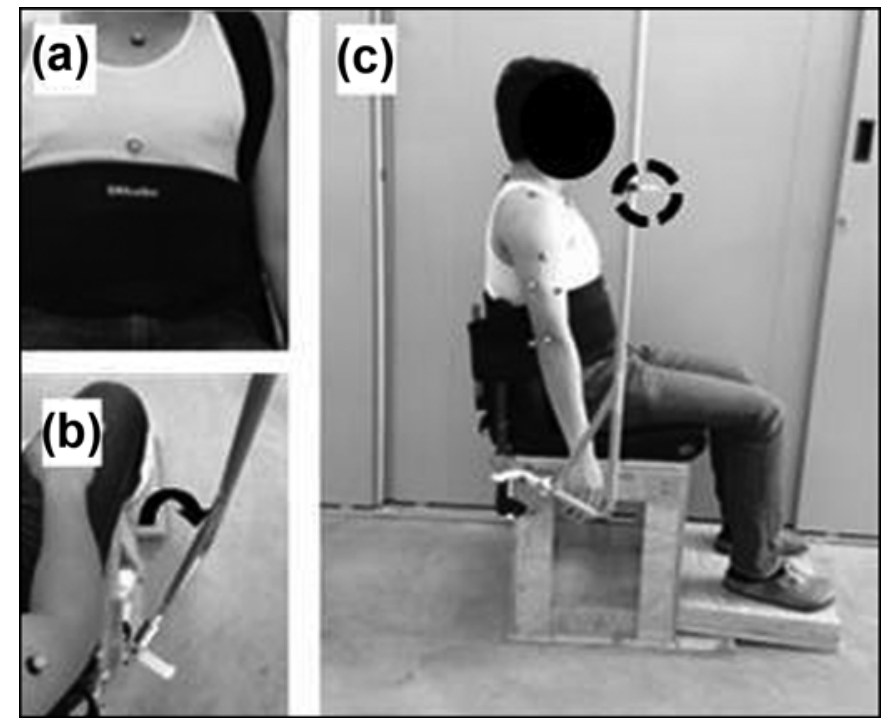

\section{Figure 2.}

(a) Testing chair and setup with trunk strap, (b) angle-adjustable guide bar, and (c) stop marker at desired arm elevation. 
have been affected by changes in trunk or arm position, we also compared trunk and arm position across trials.

\section{Ultrasound Imaging of Scapula}

One operator, with experience collecting ultrasound images of bone and soft tissue of the shoulder and upper limb in a research setting, performed all ultrasound scanning. The depth of the ultrasound imaging was set to $4 \mathrm{~cm}$ for all participants. Ample ultrasound gel was applied to the skin overlaying the scapula, and the probe was oriented to maintain visualization of the border of the scapula in the image. The ultrasound probe was moved slowly back and forth along the spine of the scapula ( $\sim 20 \mathrm{~s})$ and then up and down along the medial border of the scapula ( $\sim 40 \mathrm{~s})$. Less time was spent on the spine of the scapula because it was more superficial and easily visualized. Figure 3 illustrates different probe orientations and associated ultrasound image of the spine and medial borders of the scapula.

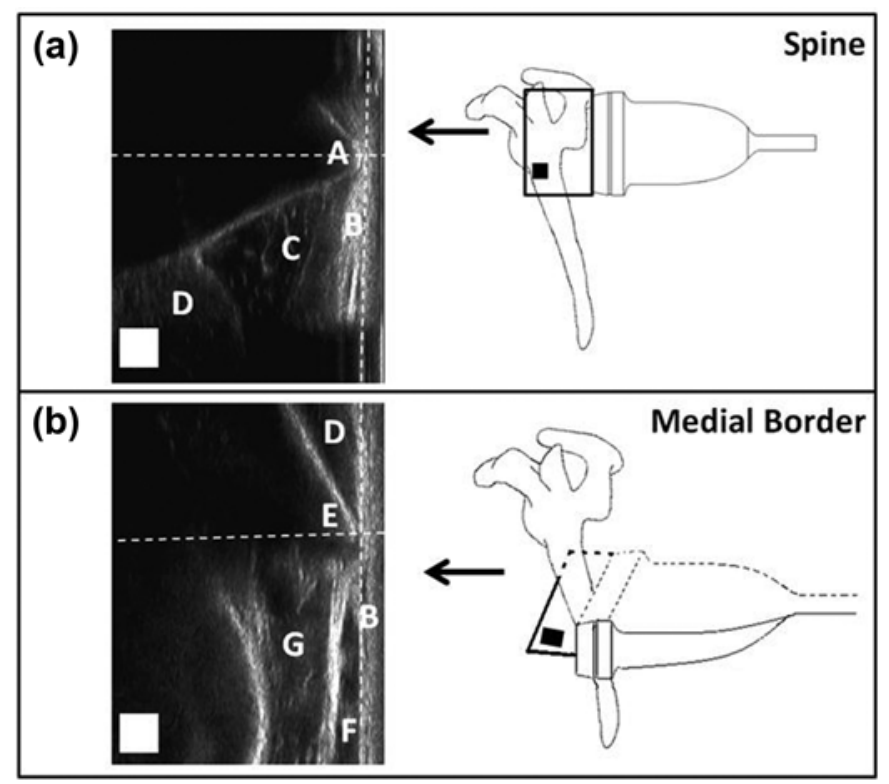

\section{Figure 3.}

(a) Imaging spine and (b) medial border of scapula with manually identified point of interest (intersection of hashed lines) and key landmarks labeled: spine (A), skin/adipose tissue (B), deltoid $(C)$, infraspinatus (D), medial border of scapula (E), trapezius (F), and rhomboid major (G). Square is located in bottom right of image as point of reference for image orientation with respect to sketch of ultrasound probe.
Participants were imaged three times in four testing positions of interest, which consisted of the arm by the participant's side at rest and humeral elevation in the sagittal, frontal, and scapular planes $\left(30^{\circ}\right.$ anterior to the frontal plane). The subject held each position for $1 \mathrm{~min}$ during scanning. A 2 min rest period was provided between all trials to prevent fatigue.

\section{Manual Point Selection Reliability}

The spine or medial border of the scapula was manually identified in each frame of the ultrasound videos. The spine of the scapula was identified as the most posterior edge while the medial border was identified as the most medial edge in the image. A reliability study was performed to determine the interrater and intrarater reliability of manual identification of the scapula in ultrasound images. The order was randomized, and raters were blinded to the trial they were viewing. Scapula identification consisted of selecting an $x-y$ coordinate in each ultrasound image representative of the scapular border. The origin of each ultrasound image was set to be the upper-left corner of the image.

\section{Data Processing}

Ultrasound videos were read into a custom MATLAB (The MathWorks; Natick, Massachusetts) program and written to individual image files. Within each image, the scapular border was manually identified as an $x-y$ coordinate. For each set of images, frames were analyzed in sequence to allow tracking of the scapula from one image to the next and prevent misidentification of the scapular border. Vicon data were exported and smoothed using a fourth-order zero-lag Butterworth filter with a cutoff frequency of $5 \mathrm{~Hz}$.

\section{Syncing Vicon and Ultrasound Systems}

Vicon and ultrasound data were synched by applying the ultrasound probe to the participant's shoulder and quickly removing it. The quick movement could be detected by an absence of ultrasound image and sharp change in movement in the location of the ultrasound probe. The sampling frequency of the frame grabber used for this study changed based on the contents of the image. To compensate for this, when Vicon and ultrasound data were combined, each second of Vicon data was spline-fit using a third-order polynomial and downsampled corresponding to the determined ultrasound sampling frequency for that envelope $(6-17 \mathrm{~Hz})$. 


\section{Reconstructing Three-Dimensional Scapular Border}

A series of transformations were applied to the 2D point identified as the scapular border in each ultrasound image $\left(P_{\text {beam }}\right)$ to determine the location of this point in 3D space $\left(P_{\text {global }}\right)$. $P_{\text {global }}$ was determined based on a static calibration relating the ultrasound beam to the orthogonal attachment ( $\left.T_{\text {beams, attachs }}\right)$, the location of the probe during testing $\left(T_{\text {attachD,global }}\right)$, the position of the subject's trunk at the time the image was collected $\left(T_{\text {global,trunkD }}\right)$, and the average trunk position of the subject during testing $\left(T_{\text {trunkS,global }}\right)$ (Equation 1):

$$
\begin{aligned}
& P_{\text {global }}=\left[T_{\text {trunkS, global }}\right]\left[T_{\text {global,trunkD }}\right] \\
& {\left[T_{\text {attachD, global }}\right]\left[T_{\text {beams, attachs }}\right] * P_{\text {beam }} .}
\end{aligned}
$$

Because trunk movement would affect scapular position, we input the position of the trunk at the time the image was collected, $T_{\text {global,trunkD }}$. This helped minimize any distortions that could be caused by the participant shifting the position of his or her torso during testing. Once the location of the scapular border was determined for each of the ultrasound images collected during the trial, the points were pooled to create a $3 \mathrm{D}$ reconstruction.

\section{Determining Scapular Position}

The International Society of Biomechanics recommends using three landmarks to determine the scapula local coordinate system: acromial angle (origin), root of the spine, and inferior angle. The z-axis extends from the root of the spine to the acromial angle [22]. The $x$-axis extends anteriorly and is perpendicular to the plane formed by the three landmarks. The $y$-axis then points upwards as the cross-product of the $x$ and $z$ axes. Because our methods involved scanning the spine and medial border of the scapula rather than identifying these three landmarks, we modified our local coordinate system to mirror methods used by the International Society of Biomechanics. We determined our z-axis as a linear regression of the points making up the spine of the scapula border, the $x$-axis as perpendicular to a plane fit to all the points in our scapular point cloud, and the $y$-axis as their cross product. The scapular position was determined with respect to the trunk, where $T$ is the transpose of the matrix (Equation 2):

$$
T_{\text {trunk,scap }}=\left(T_{\text {global,trunk }}\right)^{T} * T_{\text {global }, \text { scap }} .
$$

To determine rotations, we followed the International Society of Biomechanics' recommendation for Euler angle sequence [22]. A YXZ rotation sequence was used for matrix decomposition to determine scapular rotation, with the first rotation about the $y$-axis representing internal/ external rotation $(\alpha)$, the second about the $x$-axis upward/ downward rotation $(\beta)$, and the third about the $z$-axis anterior/posterior tilting $(\gamma)$. With this coordinate system, positive rotations included internal rotation, downward rotation, and posterior tilting (Figure 4).

\section{Determining Humeral Elevation and Trunk Position}

The humeral coordinate system was determined using the following markers: acromion, lateral epicondyle, and medial epicondyle. The $y$-axis was formed by the line extending upward from the midpoint between the medial and lateral epicondyles to the acromion. The $x$ axis was the line formed perpendicular to the plane created by the lateral and medial epicondyles and the acromion, pointing forward. The $z$-axis was determined as the

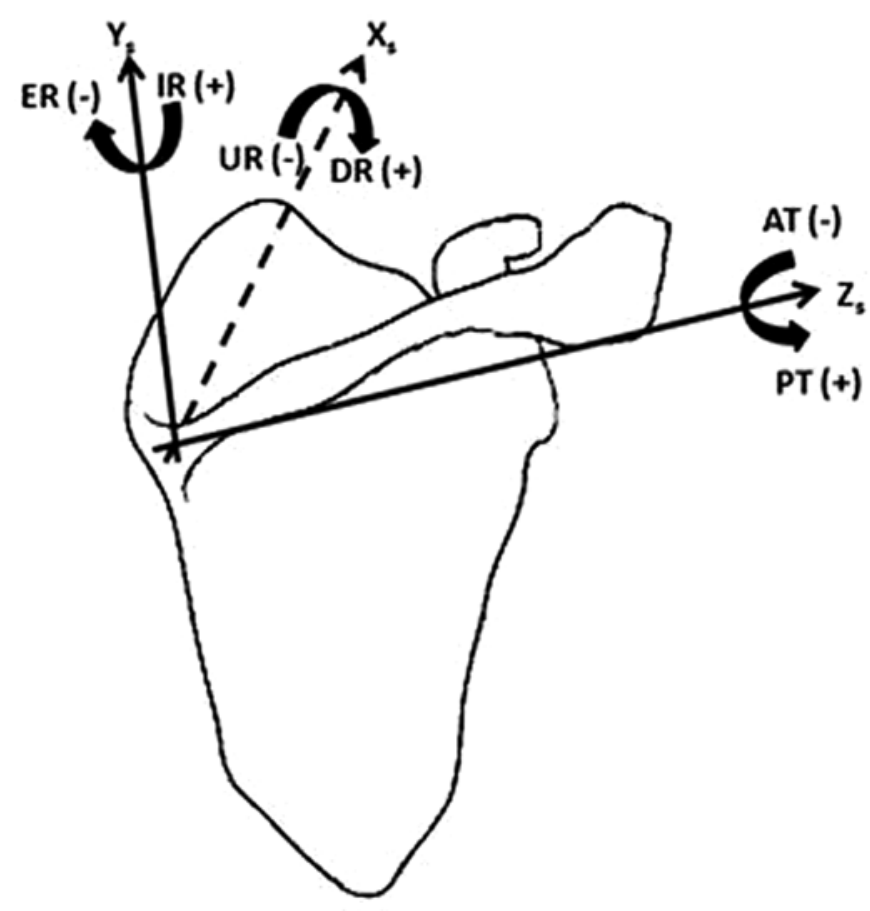

Figure 4.

Scapula coordinate system and rotations: internal/external rotation (IR/ER), upward/downward rotation (UR/DR), and anterior/posterior tilting (AT/PT). 
cross-product of the $x$ - and $y$-axes. The angle of humeral elevation was determined with respect to the trunk, where $T$ is the transpose of the matrix (Equation 3):

$$
T_{\text {trunk,humerus }}=\left(T_{\text {global,trunk }}\right)^{T} * T_{\text {global, humerus }} \text {. }
$$

A YXY rotation sequence was used for matrix decomposition to determine humeral elevation. The sequence of rotations was elevation in the glenohumeral plane, negative glenohumeral elevation, and glenohumeral axial rotation. Trunk position was determined using a ZXY matrix decomposition of the trunk local coordinate system. The first rotation was flexion/extension, followed by lateral rotation, and axial rotation.

\section{Statistical Analysis}

To evaluate the interrater and intrarater reliability of manual point detection, intraclass correlation coefficients (ICCs) were calculated for single measures using twoway random model, $\operatorname{ICC}(2,1)$. The ICC was interpreted using the categories of agreement suggested by Landis and Koch [23], in which $\leq 0.40$ is unacceptable, 0.410.60 is moderate, $0.61-0.80$ is substantial, and $\geq 0.81$ is almost perfect. ICCs were also used to evaluate the consistency in measured scapular rotations across repeated trials. The ICC for single measures two-way mixed model, $\operatorname{ICC}(3,1)$, was calculated for each rotation of the scapula in each of the testing positions (rest and elevation in each of the three planes). Equations $\mathbf{4}$ and $\mathbf{5}$ were used to calculate standard error of measurement (SEM) and minimum detectable change (MDC), respectively:

$$
\begin{gathered}
\text { SEM }=\text { SDpooled } * \sqrt{(1-\operatorname{ICC}(3,1))} \text { and } \\
M D C=S E M * \sqrt{2} * 1.64 .
\end{gathered}
$$

To compare trunk and arm position between repeated trials, a repeated measures analysis of variance was completed for both measures with a within-subjects factor of trial and testing position (rest and elevation in the sagittal, frontal, and scapular planes). All statistical analyses were completed with IBM SPSS Statistics Software, version 20 (IBM Corporation; Armonk, New York), with the significance level set a priori at 0.05 .

\section{RESULTS}

Twenty-two healthy, nondisabled individuals (16 male, 6 female; mean \pm standard deviation age $=50.5 \pm$ $11.6 \mathrm{yr}$, height $=1.72 \pm 0.13 \mathrm{~m}$, body mass $=74.22 \pm$ $15.02 \mathrm{~kg}$ ) participated in this study approved by the University of Pittsburgh and Department of Veterans Affairs (VA) Pittsburgh Healthcare System Institutional Review Board. Consent of each subject was obtained prior to the study. For manual identification of the scapular border in ultrasound images, we found inter- and intrarater reliabilities ranging from 0.975 to 0.995 . An example of the reconstructed scapular border for one subject in each testing position can be seen below in Figure 5. A summary of scapular position across subjects in each testing position can be found in Table 1. No significant differences were found across repeated trials in any testing plane for trunk $(p=0.33-0.90)$ or arm position $(p=0.28-0.54)$.

A summary of reliability, SEM, and MDC for scapular rotations between trials can be found in Table 1. Reliability across trials ranged from substantial to almost perfect. The SEM was less than $2^{\circ}$ for all trials and can be seen in Table 2. SEM was less than $0.5^{\circ}$ for internal/ external rotation and anterior/posterior tilting for all testing positions. SEM was highest for upward/downward rotation, specifically in the sagittal and frontal planes. Because MDC is related to reliability and SEM, it is not surprising that the smallest MDC was also found when the arm was in the rest position and for internal/external rotation. For testing in the elevated positions, the MDC for internal/external rotation and upward/downward tilting was considerably smaller than the MDC for upward/ downward rotation.

We found no significant differences across trials for arm or trunk position across repeated trials. It is important to note that the average arm elevation angle, measured based on Vicon markers with respect to the trunk, for subjects in the elevated trials did not reach $120^{\circ}$, but instead was closer to $90^{\circ}$ (Table 2).

\section{DISCUSSION}

The objective of this study was to evaluate the reliability of using freehand 3D ultrasound to determine scapular rotations. Very high intrarater reliability was found for all testing positions and all scapular rotations across trials. The greatest reliability was found in the 


\begin{tabular}{|l|l|l|l|l|}
\hline Scapula & Sest & Sagittal Plane Elevation & Frontal Plane Elevation & Scapular Plane Elevation \\
\hline & & & & \\
\hline
\end{tabular}

Figure 5.

Example of reconstructed scapula in all testing positions (sagittal plane $[x-y]$ top row, transverse plane $[x-z]$ bottom row).

Table 1.

Scapular rotations across testing positions. Data shown as mean \pm standard deviation.

\begin{tabular}{lccrr}
\hline \multirow{2}{*}{ Scanning Position } & \multicolumn{4}{c}{ Scapular Rotation } \\
\cline { 2 - 5 } & $\begin{array}{c}\text { Arm Elevation } \\
\text { Angle ( }\left(^{\circ}\right.\end{array}$ & $\begin{array}{c}\text { Internal (+)/External (-) } \\
\text { Rotation ( }\left(^{\circ}\right)\end{array}$ & $\begin{array}{c}\text { Upward (-)/Downward (+) } \\
\text { Rotation ( }\left(^{\circ}\right)\end{array}$ & $\begin{array}{c}\text { Anterior (-)/Posterior (+) } \\
\text { Tilting }\left(^{\circ}\right)\end{array}$ \\
\hline Rest & $17.8 \pm 5.8$ & $31.6 \pm 6.4$ & $-0.5 \pm 8.5$ & $-12.4 \pm 5.9$ \\
Sagittal Plane Elevation & $90.5 \pm 11.5$ & $34.9 \pm 9.5$ & $-36.4 \pm 6.9$ & $3.1 \pm 5.2$ \\
Frontal Plane Elevation & $89.9 \pm 10.5$ & $21.8 \pm 8.9$ & $-40.8 \pm 6.6$ & $4.3 \pm 7.1$ \\
Scapular Plane Elevation & $87.4 \pm 11.8$ & $29.0 \pm 8.5$ & $-37.6 \pm 6.6$ & $2.9 \pm 6.3$ \\
\hline \hline
\end{tabular}

Table 2.

Intraclass correlation coefficient (ICC), standard error of measurement (SEM), and minimum detectable change (MDC) for repeated freehand three-dimensional ultrasound scans.

\begin{tabular}{|c|c|c|c|c|c|c|c|c|c|}
\hline \multirow{3}{*}{ Scanning Position } & \multicolumn{9}{|c|}{ Scapular Rotation $(n=22)$} \\
\hline & \multicolumn{3}{|c|}{ Internal/External Rotation $\left(^{\circ}\right)$} & \multicolumn{3}{|c|}{ Upward/Downward Rotation $\left(^{\circ}\right)$} & \multicolumn{3}{|c|}{ Anterior/Posterior Tilting $\left(^{\circ}\right)$} \\
\hline & ICC (95\% CI) & SEM & MDC & ICC (95\% CI) & SEM & MDC & ICC (95\% CI) & SEM & MDC \\
\hline$\overline{\text { Rest }}$ & $0.95(0.89-0.98)$ & 0.16 & 0.37 & $0.89(0.79-0.95)$ & 0.47 & 1.08 & $0.92(0.84-0.97)$ & 0.19 & 0.44 \\
\hline Sagittal Plane & $0.93(0.86-0.97)$ & 0.34 & 0.79 & $0.69(0.48-0.84)$ & 1.14 & 2.66 & $0.87(0.76-0.94)$ & 0.37 & 0.86 \\
\hline Frontal Plane & $0.94(0.82-0.97)$ & 0.25 & 0.57 & $0.62(0.38-0.81)$ & 1.33 & 3.08 & $0.94(0.88-0.97)$ & 0.21 & 0.49 \\
\hline Scapular Plane & $0.93(0.86-0.97)$ & 0.27 & 0.62 & $0.80(0.64-0.90)$ & 0.65 & 1.52 & $0.90(0.80-0.95)$ & 0.32 & 0.74 \\
\hline
\end{tabular}

resting position. This is not surprising because elbow flexion and wrist flexion could possibly vary across trials in the elevated testing positions, thus affecting arm position and, ultimately, scapular position. Additionally, the rest position required no effort on the part of the participant to maintain.
Ludewig et al. investigated reliability of landmark digitization for humeral elevation in the scapular plane to determine scapular rotations and found ICCs of 0.93 for internal/external rotation, 0.82 for upward/downward rotation, and 0.78 for anterior/posterior tilting [24]. We had ICCs that were equal to or exceeded the reliability of 
their study. Bourne et al. also completed a reliability study on the use of bone pins for determining scapular position during elevation in the sagittal and frontal planes [6]. They found ICCs that exceeded 0.85 for internal/ external rotation, 0.97 for upward/downward rotation, and 0.97 for anterior/posterior tilting. Comparatively, our reliability exceeds that of Bourne et al. for internal/external rotation. Reliability for other rotations is not as high as that found by Bourne et al.; however, our study still shows substantial to excellent reliability. This finding is significant when acknowledging that while bone pins are reliable, they are also invasive.

Many other studies do not report SEM for scapular rotation measurements. Ludewig et al. reported SEM for the scapular plane of less than $2^{\circ}$ for anterior/posterior tilting and less than $3^{\circ}$ for both internal/external rotation and upward/downward rotation [24]. Our SEM was less than $2^{\circ}$ for all rotations in all testing positions. Bourne et al. did not report SEM but did report RMSE ranging from $1.2^{\circ}$ to $4^{\circ}$. Additionally, MDC for scapular movement has also not been reported by previous studies. Because MDC is a function of SEM, we can compare it to the study by Ludewig et al. With their reported range of SEM from $2^{\circ}$ to $3^{\circ}$, the resulting $\mathrm{MDC}_{90}$ would be $4.6^{\circ}-7.0^{\circ}$. Comparatively, our MDC at $90^{\circ}$ elevation is lower at less than $1^{\circ}$ for internal/external rotation and anterior/posterior and approximately $3^{\circ}$ or less for upward/downward rotation in all positions. We found good agreement for scapular position at rest with previous studies for internal and upward rotation [10,24-26]. In general, we found increased external rotation, upward rotation, and posterior tilting with humeral elevation, which is what we would have expected [6].

The results of this study are based on a relatively small $(n=22)$ sample of healthy, nondisabled individuals. To apply these methods to future studies, it is also important to evaluate the reliability with other populations of interest. Body composition has an effect on the quality of ultrasound imaging because significant adipose tissue or muscle mass can affect impedance. All participants in this study had a body mass index of less than 25; therefore, imaging may have been easier. As previously indicated, there were variations associated with the sampling frequency of the frame grabber used to collect ultrasound data in this study. To compensate for this variable rate, we resampled our data based on an approximate rate determined by the timestamp on ultrasound images. Inherently, this introduces additional error into our calcu- lations because the approximation cannot make up for the asynchrony in the time domain. While high reliability was still found between trials, future studies that avoid use of devices with dynamic sampling may have higher reliability.

An additional limitation of this study is that we did not collect data using a gold standard measure, like bone pins. While incorporation of a gold standard would have allowed for direct comparison, bone pins are invasive and would have significantly limited our sample size. However, without this gold standard, we are unable to make direct comparisons between our results and existing methods. Future studies should include a subsample evaluated with a gold standard or should use other forms of simultaneous measurement, such as radiograph imaging, to compare our results. Additionally, future studies should incorporate interrater reliability using different ultrasound operators to validate our methodology. Our technique could be used for dynamic measurement during movement, and this, too, should be investigated in future studies. On the basis of these preliminary results using ultrasound to evaluate static postures, we believe that the ability to visualize and reconstruct scapular borders would lend itself well to providing a measure to detect scapular dyskinesia.

\section{CONCLUSIONS}

Freehand 3D ultrasound shows promise as a tool for evaluating scapular positioning. We were able to apply this method to a variety of different testing positions and achieved high intrarater reliability in all positions. Additionally, our SEM was less than $2^{\circ}$ for all measurements and less than $0.5^{\circ}$ for most. Our results agree with the pattern of movement found in other studies, with the scapula moving toward a more externally rotated, upwardly rotated, and posterior tilted position. Because body composition affects the ultrasound image, it is important to be aware of the limitations imposed on this method by the presence of large amounts of adipose tissue or muscle mass. The frame grabber used in this study was the best technology available to us at the time of the study, but results may improve with ongoing advancements in ultrasound video recording and sampling techniques. We plan on incorporating a gold standard and dynamic measurement to future studies to evaluate 
whether these methods are sensitive to detecting pathology such as scapular dyskinesia.

\section{ACKNOWLEDGMENTS}

\section{Author Contributions:}

Study concept and design: L. A. Worobey, S. S. Farrokhi, M. L. Boninger.

Analysis and interpretation of data: L. A. Worobey, Y. Lin, A. M. Koontz, M. L. Boninger.

Drafting of manuscript: L. A. Worobey, I. A. Udofa.

Critical revision of manuscript for important intellectual content:

L. A. Worobey, I. A. Udofa, Y. Lin, A. M. Koontz, S. S. Farrokhi,

M. L. Boninger.

Financial Disclosures: The authors have declared that no competing interests exist.

Funding/Support: This material was based on work supported by the VA Center for Excellence for Wheelchairs and Associated Rehabilitation Engineering (grant B6789C) and a National Science Foundation Graduate Research Fellowship. This material is also the result of work supported with resources and the use of facilities at the Human Engineering Research Laboratories, VA Pittsburgh Healthcare System. Institutional Review: This study was approved by the University of Pittsburgh and VA Pittsburgh Healthcare System Institutional Review Board. Consent of each subject was obtained prior to the study. Participant Follow-Up: The authors do not plan to inform participants of the publication of this study. However, participants have been encouraged to check the laboratory Web site for updated publications. Additionally, our laboratory newsletter provides information on recently published articles.

\section{REFERENCES}

1. Fu FH, Harner CD, Klein AH. Shoulder impingement syndrome. A critical review. Clin Orthop Relat Res. 1991; (269):162-73. [PMID:1864035]

2. Ludewig PM, Cook TM. Alterations in shoulder kinematics and associated muscle activity in people with symptoms of shoulder impingement. Phys Ther. 2000;80(3):276-91. [PMID:10696154]

3. Lukasiewicz AC, McClure P, Michener L, Pratt N, Sennett B. Comparison of 3-dimensional scapular position and orientation between subjects with and without shoulder impingement. J Orthop Sports Phys Ther. 1999;29(10): 574-83, discussion 584-86. [PMID:10560066] http://dx.doi.org/10.2519/jospt.1999.29.10.574

4. McClure PW, Michener LA, Karduna AR. Shoulder function and 3-dimensional scapular kinematics in people with and without shoulder impingement syndrome. Phys Ther. 2006;86(8):1075-90. [PMID:16879042]

5. Warner JJ, Micheli LJ, Arslanian LE, Kennedy J, Kennedy R. Scapulothoracic motion in normal shoulders and shoul- ders with glenohumeral instability and impingement syndrome. A study using Moiré topographic analysis. Clin Orthop Relat Res. 1992;(285):191-99. [PMID:1446436]

6. Bourne DA, Choo AM, Regan WD, MacIntyre DL, Oxland TR. Three-dimensional rotation of the scapula during functional movements: An in vivo study in healthy volunteers. J Shoulder Elbow Surg. 2007;16(2):150-62.

[PMID:17113324]

http://dx.doi.org/10.1016/j.jse.2006.06.011

7. McClure PW, Michener LA, Sennett BJ, Karduna AR. Direct 3-dimensional measurement of scapular kinematics during dynamic movements in vivo. J Shoulder Elbow Surg. 2001;10(3):269-77. [PMID:11408911] http://dx.doi.org/10.1067/mse.2001.112954

8. van Andel C, van Hutten K, Eversdijk M, Veeger D, Harlaar J. Recording scapular motion using an acromion marker cluster. Gait Posture. 2009;29(1):123-28. [PMID: 18815043] http://dx.doi.org/10.1016/j.gaitpost.2008.07.012

9. Brochard S, Lempereur M, Rémy-Néris O. Accuracy and reliability of three methods of recording scapular motion using reflective skin markers. Proc Inst Mech Eng H. 2011; 225(1):100-105. [PMID:21381493] http://dx.doi.org/10.1243/09544119JEIM830

10. Johnson GR, Stuart PR, Mitchell S. A method for the measurement of three-dimensional scapular movement. Clin Biomech (Bristol, Avon). 1993;8(5):269-73.

[PMID:23915988] http://dx.doi.org/10.1016/0268-0033(93)90037-I

11. Finley MA, Rodgers MM. Prevalence and identification of shoulder pathology in athletic and nonathletic wheelchair users with shoulder pain: A pilot study. J Rehabil Res Dev. 2004;41(3B):395-402. [PMID:15543457] http://dx.doi.org/10.1682/JRRD.2003.02.0022

12. Meskers CG, Vermeulen HM, de Groot JH, van Der Helm FC, Rozing PM. 3D shoulder position measurements using a six-degree-of-freedom electromagnetic tracking device. Clin Biomech (Bristol, Avon). 1998;13(4-5):280-92.

[PMID:11415798] http://dx.doi.org/10.1016/S0268-0033(98)00095-3

13. Bourne D, Choo A, Regan W, MacIntyre D, Oxland T. Accuracy of digitization of bony landmarks for measuring change in scapular attitude. Proc Inst Mech Eng H. 2009; 223(3):349-61. [PMID:19405440] http://dx.doi.org/10.1243/09544119JEIM480

14. Farris DJ, Trewartha G, McGuigan MP, Lichtwark GA. Differential strain patterns of the human Achilles tendon determined in vivo with freehand three-dimensional ultrasound imaging. J Exp Biol. 2013;216(4):594-600.

[PMID:23125339] http://dx.doi.org/10.1242/jeb.077131 
15. Cronin NJ, Lichtwark G. The use of ultrasound to study muscle-tendon function in human posture and locomotion. Gait Posture. 2013;37(3):305-12. [PMID:22910172] http://dx.doi.org/10.1016/j.gaitpost.2012.07.024

16. Lichtwark GA, Wilson AM. In vivo mechanical properties of the human Achilles tendon during one-legged hopping. J Exp Biol. 2005;208(24):4715-25. [PMID:16326953] http://dx.doi.org/10.1242/jeb.01950

17. Bénard MR, Harlaar J, Becher JG, Huijing PA, Jaspers RT. Effects of growth on geometry of gastrocnemius muscle in children: A three-dimensional ultrasound analysis. J Anat. 2011;219(3):388-402. [PMID:21635250] http://dx.doi.org/10.1111/j.1469-7580.2011.01402.x

18. Rana M, Wakeling JM. In-vivo determination of 3D muscle architecture of human muscle using free hand ultrasound. J Biomech. 2011;44(11):2129-35.

[PMID:21664617]

http://dx.doi.org/10.1016/j.jbiomech.2011.05.026

19. Weller R, Pfau T, Ferrari M, Griffith R, Bradford T, Wilson A. The determination of muscle volume with a freehand 3D ultrasonography system. Ultrasound Med Biol. 2007; 33(3):402-7. [PMID:17208353] http://dx.doi.org/10.1016/j.ultrasmedbio.2006.08.007

20. Monk AP, Chen M, Gibbons CLMH, Beard DJ, Gill HS, Murray D. Measurement of in-vivo patella kinematics using motion analysis and ultrasound (MAUS). Proceedings of the IEEE International Symposium on Medical Measurements and Applications; 2013 May 4-5; Gatineau, Quebec, Canada.

21. Monk AP, Chen M, Mellon S, Gibbons M, Beard DJ, Murray DW, Gill HS. Comparison of in vivo coronal plane patella tracking following knee arthroplasty using the MAUS technique. Bone Joint J Orthop Proc Suppl. 2013; 95(Supp 26): 10 .

22. Wu G, van der Helm FC, Veeger HE, Makhsous M, Van Roy P, Anglin C, Nagels J, Karduna AR, McQuade K, Wang X, Werner FW, Buchholz B; International Society of
Biomechanics. ISB recommendation on definitions of joint coordinate systems of various joints for the reporting of human joint motion-Part II: shoulder, elbow, wrist and hand. J Biomech. 2005;38(5):981-92. [PMID:15844264] http://dx.doi.org/10.1016/j.jbiomech.2004.05.042

23. Landis JR, Koch GG. The measurement of observer agreement for categorical data. Biometrics. 1977;33(1):159-74. [PMID: 843571]

24. Ludewig PM, Cook TM, Nawoczenski DA. Three-dimensional scapular orientation and muscle activity at selected positions of humeral elevation. J Orthop Sports Phys Ther. 1996;24(2):57-65. [PMID:8832468] http://dx.doi.org/10.2519/jospt.1996.24.2.57

25. Culham E, Peat M. Functional anatomy of the shoulder complex. J Orthop Sports Phys Ther. 1993;18(1):342-50. [PMID:8348135] http://dx.doi.org/10.2519/jospt.1993.18.1.342

26. Laumann U. Kinesiology of the shoulder joint. In: Koelbel R, Helbig B, Blauth W. Shoulder replacement. Berlin (Germany): Springer-Verlag; 1987. p. 23-31.

Submitted for publication January 9, 2014. Accepted in revised form March 6, 2014.

This article and any supplementary material should be cited as follows:

Worobey LA, Udofa IA, Lin Y, Koontz AM, Farrokhi SS, Boninger ML. Reliability of freehand three-dimensional ultrasound to measure scapular rotations. J Rehabil Res Dev. 2014;51(6):985-94.

http://dx.doi.org/10.1682/JRRD.2014.01.0006

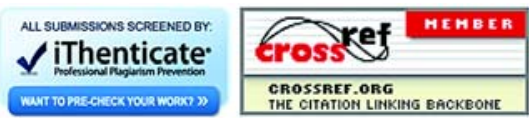

